\title{
Spinal Teratoma Concomitant with Intracranial Lipid Droplet Dissemination
}

\author{
Hyung Sug Oh, Tae Wan Kim, Kwan Ho Park \\ Department of Neurosurgery, VHS Medical Center, Seoul, Korea
}

\begin{abstract}
A teratoma is a neoplasm that contains tissues originating from three germ cell layers at ectopic sites. The embryology of teratomas remains unclear. Teratomas are usually composed of cystic and solid components, and they are usually associated with syringomyelia. Cystic lesions of teratomas may rupture in a spontaneous, iatrogenic, or traumatic manner. Lipid droplets in the ventricles and subarachnoid space are rare. We managed a case of a spinal teratoma in the lumbar region in a 67-year-old man. He complained of nocturia, frequent urination, and difficulty in walking for 2 months. Radiographic imaging revealed a lumbar spinal intradural mass. Intracranial lipid droplets dissemination was also existed. The patient underwent surgery, and a diagnosis of mature teratoma was confirmed histopathologically. During the operation, the cystic portion of the intradural mass ruptured. During the hospital stay, the patient's mental status declined. On radiological examination, slightly enlarged ventricle size was observed. Dissemination of lipid droplets within ventricles occurs because of spontaneous, iatrogenic, or traumatic rupture. Additional lipid droplet dissemination to the intracranial space associated with neurologic deterioration after a spinal teratoma surgery should be considered when iatrogenic rupture of the cyst portion occurs.
\end{abstract}

Key Words: Teratoma $\cdot$ Spine $\cdot$ Intracranial $\cdot$ Lipid droplet

\section{INTRODUCTION}

Spinal cord teratomas are uncommon. Excluding sacrococcygeal teratoma in neonates, teratomas in the spinal canal are rare. Only a few central nervous system (CNS) teratomas arise from the spinal cord. The clinical symptoms may be secondary to mechanical cord compression. Diagnostic confirmation of teratomas depends on pathological findings. Delayed presentation of spinal teratomas has generally been associated with its slow-growing characteristics).

Dermoid tumors may rupture and fatty material may escape into the subarachnoid space, and/or ventricles, resulting in variable neurological symptoms ${ }^{6}$. The Symptoms are dependent on location and are due to the irritative effect on and/or compression of the adjacent structures ${ }^{3)}$. The tumors may be asymptomatic but can result in coma or death. Aseptic menin-

- Received: October 16, 2014 - Revised: January 18, 2015

- Accepted: February 4, 2015

Corresponding Author: Tae Wan Kim, MD

Department of Neurosurgery, VHS Medical Center, 53 Jinhwangdo-ro,

61 -gil, Gangdong-gu, Seoul 134-791, Korea

Tel: +82-2-2225-1363, Fax: +82-2-2225-4152

E-mail: euro3399@naver.com

œThis is an Open Access article distributed under the terms of the Creative

Commons Attribution Non-Commercial License (http://creativecommons.org/

licenses/by-nc/3.0/) which permits unrestricted non-commercial use, distribution

and reproduction in any medium, provided the original work is properly cited. gitis may also occur ${ }^{15)}$.

Here, we report a rare case of a mature intradural spinal teratoma in an adult with concomitant additional intracranial lipid droplet dissemination associated with neurologic deterioration after iatrogenic rupture of the cyst portion occurs. Iatrogenic rupture of cystic portion during spinal teratoma surgery may worsen intracranial lesion that previously exist.

\section{CASE REPORT}

A 67-year-old man was transferred to our department from the urology department of our hospital. He complained of nocturia and frequent urination. During evaluation, an approximately $3 \mathrm{~cm}$ mass in the spinal canal was detected incidentally on abdominal computed tomography (CT) imaging. Abdominal CT imaging showed a fat-containing mass with mural calcification at the L2 level (Fig. 1). The patient looked ill and had experienced aplastic anemia 2 years previously. On neurologic examination, an approximately grade 4 weakness was noted in both lower extremities. He could not walk without support since the last 2 months. Lumbar magnetic resonance (MR) imaging showed a hyperintense intradural mass on a T1-weighted image. After gadolinium enhancement, there was no significant enhancement of the mass in the conus medullaris and focal enhancement of the distal soft tissue component (Fig. 2). He underwent total laminectomy and tumor resection. 
During the operation, the cystic portion of the intradural mass was ruptured. After surgery, his lower-extremity weakness did not improve, and he could not walk without assistance. Histopathologically, a mature teratoma was confirmed (Fig. 3). After surgery, his general condition worsened gradually. Two weeks after surgery, his mental status also deteriorated. Preoperative CT imaging showed low density lesion in both lateral ventricle and cistern. At that time, he didn't complain any symptom. Postoperative brain MR imaging showed hyperintense lesion on a T1-weighted image on both the lateral ventricle and as well as slightly enlarged ventricles (Fig. 4). We performed extra ventricular drainage (EVD), and his mental status recovered temporarily. However, his mental status again declined; EVD was performed on the other side after 7 days but, we could not retrieve tissue from the mass. During treatment, his general condition and mental status worsened further. Finally, he died of pneumonia and sepsis.

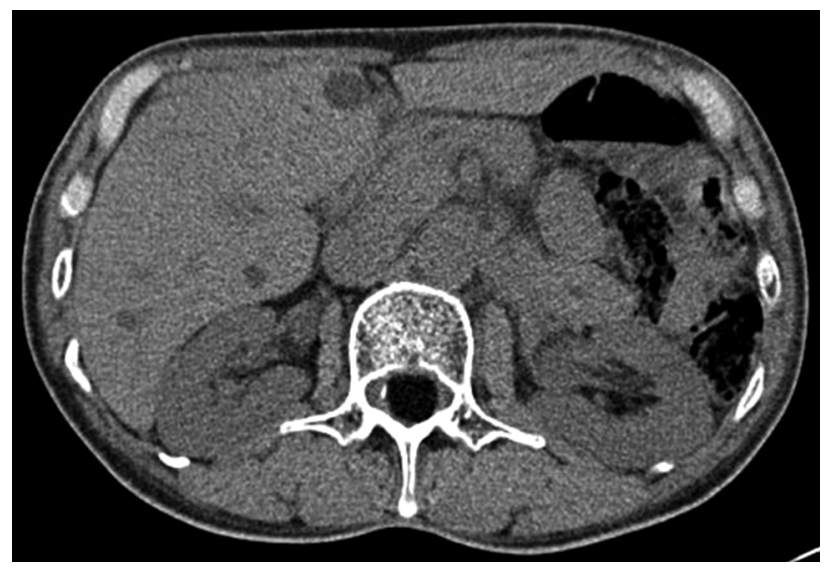

Fig. 1. Non-enhancing abdominal computed tomography image showing a hypodense round mass in the spinal canal.

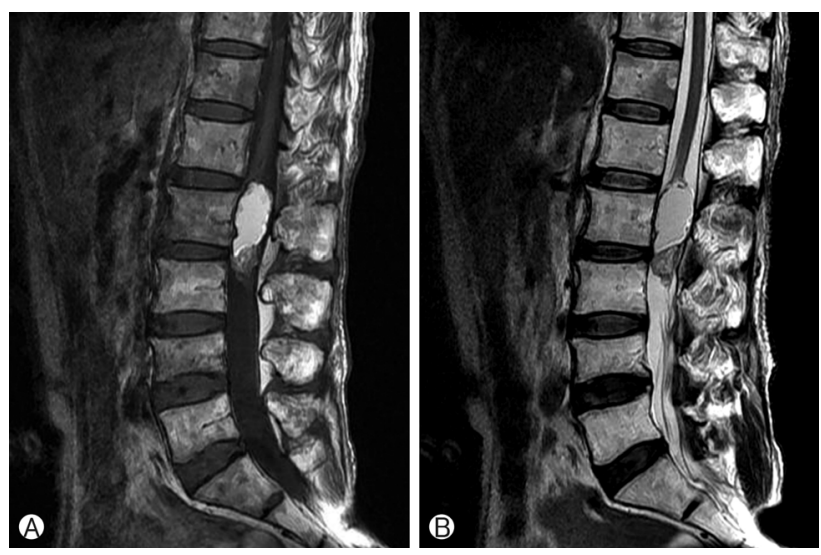

Fig. 2. Sagittal T1-weighted (A) and T2-weighted (B) MR images showing a hyperintense spindle mass with a peripheral heterogeneous mixed-signal lesion.

\section{DISCUSSION}

Spinal cord teratomas are uncommon. Only a few CNS teratomas arise from the spinal cord. Epidural spinal teratomas are rarer than intradural spinal teratomas ${ }^{14)}$. Teratomas are benign germ cell tumors that are slow growing in nature. They arise in ectopic sites and are composed of multiple embryologic layers. Teratomas may be composed of mature or immature tissues. Teratoids are composed of poorly diffentiated structures or elements from only two germinal layers. The prognosis of teratoids is poor and metastases at diagnosis are common, especially in neonates and children ${ }^{4}$.
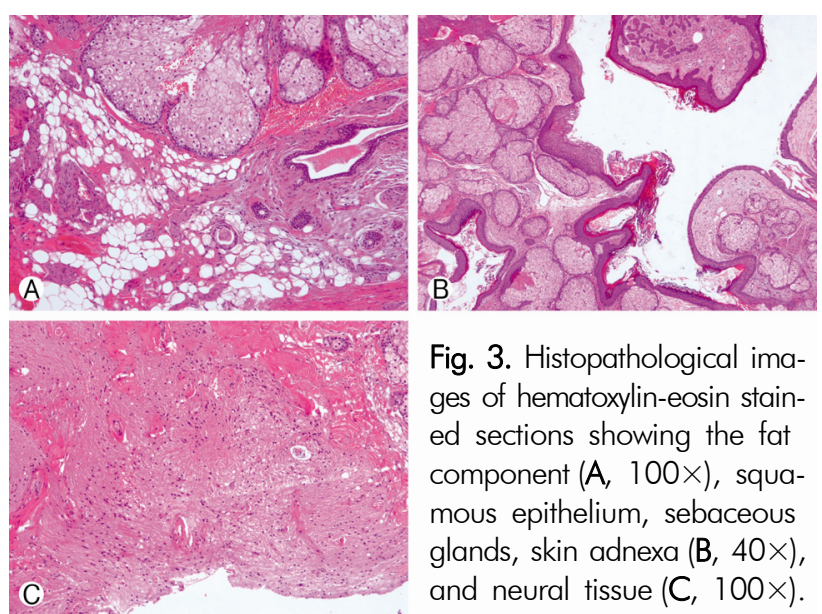

Fig. 3. Histopathological images of hematoxylin-eosin stained sections showing the fat component (A, 100X), squamous epithelium, sebaceous glands, skin adnexa $(B, 40 \times)$, and neural tissue $(C, 100 \times)$.
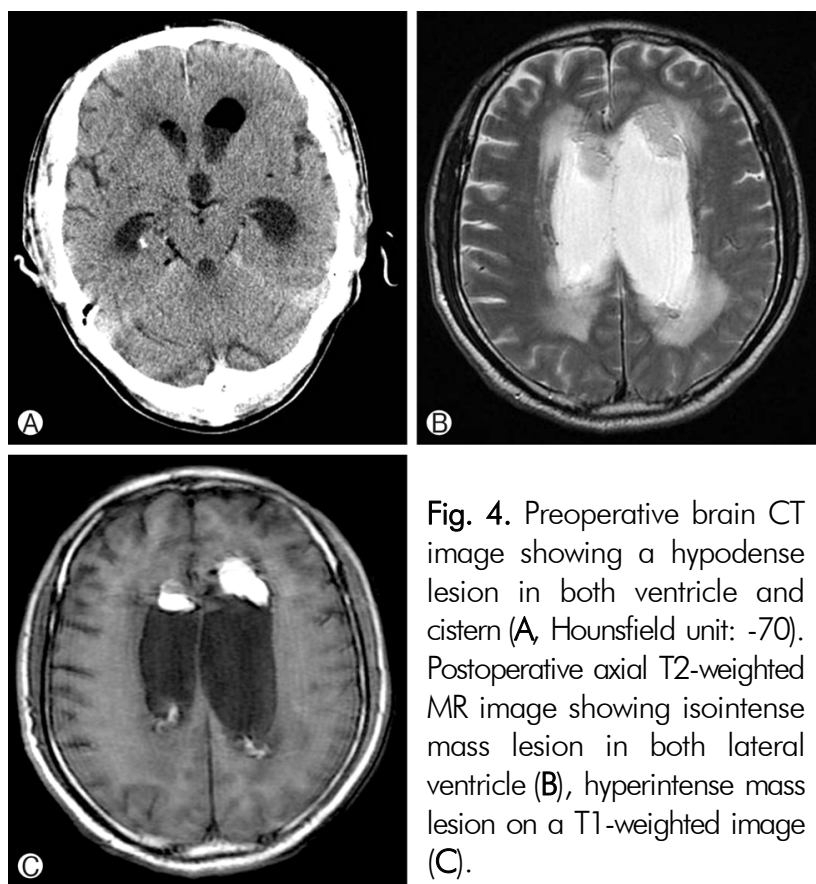

Fig. 4. Preoperative brain CT image showing a hypodense lesion in both ventricle and cistern (A, Hounsfield unit: -70). Postoperative axial T2-weighted MR image showing isointense mass lesion in both lateral ventricle (B), hyperintense mass lesion on a $\mathrm{Tl}$-weighted image (C). 
Teratomas are slow growing, but, often grow outside the spinal column and extend to the paraspinal and retroperitoneal spaces. Mature teratomas are composed of well-differentiated elements, and immature teratomas are primitive elements derived from any or all of the three germ cell layers ${ }^{2}$.

$\mathrm{MR}$ imaging is considered as the gold standard for the detection of teratomas. Contrast-enhanced imaging shows minimal and peripheral enhancement ${ }^{8,9)}$. Our case also showed a distal peripherally enhancing pattern.

Surgery is the treatment of choice for teratomas. Total resection is desirable; however, it is almost impossible because of the intimal adhesion between the teratomatous cyst and the circumferential nervous parenchyma. Radical surgery is not recommended as the pathology is benign and recurrence rates are low even after subtotal resections ${ }^{1,11}$. The surgical goal is subtotal resection for decompressing the cord and resecting the exophytic component ${ }^{5}$, and excised tissues should be taken as extensively as possible to establish an accurate diagnosis ${ }^{7}$. Recurrence after total resection of immature teratomas has been reported ${ }^{12}$. After subtotal resection, consecutive and longterm radiological follow-up is recommended ${ }^{16}$.

A case of spinal mature teratoma accompanying the intracranial dissemination of fatty droplets has been reported ${ }^{3,6,13,15)}$. Dissemination of lipid droplets within ventricles occurs because of spontaneous, iatrogenic, or traumatic rupture. Our case is similar to this previous case, although, an intracranial mass lesion was not confirmed histopathologically. The symptoms of intracranial lipid droplet dissemination were headache, chemical meningitis, seizure and mental deterioration.

The cystic contents gain access to the ventricular system by way of retrograde flow through the foramen of Luschka and Magendie ${ }^{15}$. Lipid droplets can spread throughout the subarachnoid space and ventricular system. It is possible that the central canal can serve as a pathway for ruptured material into the brain $^{13)}$.

$\mathrm{CT}$ and MR imaging are useful in diagnosing free fat in the subarachnoid space and intraventricular fat-fluid level. The Hounsfield unit is also useful in differentiating lipid and a pneumocephalus. Lipid is differentiated from cerebrospinal fluid by negative values (-20 to -130 Hounsfield units) ${ }^{15}$. Our preoperative CT also reveal fat nature mass using Hounsfield unit (-70). MR imaging is reliable in the evaluation of the presence of rupture and its extent in the subarachnoid spaces or into the central spinal canal ${ }^{10)}$. MR images utilizing T1weighted image reveal high signal intensity. Our case also shows hyperintense mass lesion in both lateral ventricles on T1-weighted image.

\section{CONCLUSION}

We report a rare case of a mature intradural spinal teratoma and concomitant intracranial lipid droplet dissemination in an adult. Additional lipid droplet dissemination to intracranial space with neurologic deterioration after a spinal teratoma surgery should be considered when iatrogenic rupture of the cyst portion occurs. It might develop the symptom and worsen hydrocephalus, although asymptomatic lipid droplets dissemination was previously existed before lumbar spine surgery.

\section{REFERENCES}

1. Ak H, Ulu MO, Sar M, Albayram S, Aydin S, Uzan M: Adult intramedullary mature teratoma of the spinal cord: review of the literature illustrated with an unusual example. Acta Neurochir 148:663-669, 2006

2. Arai Y, Takahashi M, Takeda K, Shitoto K: Adult-onset intradural spinal teratoma in the lumbar spine: A case report. J Orthop Surg 8:69-74, 2000

3. Calabro F, Capellini C, Jinkins JR: Rupture of spinal dermoid tumors with spread of fatty droplets in the cerebrospinal fluid pathways. Neuroradiology 42:572-579, 2000

4. Dufour C, Beaugrand A, Le Deley MC, Bourdeaut F, Andre $\mathrm{N}$, Leblond $\mathrm{P}$, et al: Clinicopathologic prognostic factors in childhood atypical teratoid and rhabdoid tumor of the central nervous system. Cancer 118:3812-3821, 2012

5. Ghostine S, Perry E, Vaynman S, Raghavan R, Tong KA, Samudrala S, et al: The rare case of an intramedullary cervical spinal cord teratoma in an elderly adult. Spine 34:E973-E978, 2009

6. Goyal A, Singh D, Singh AK, Gupta V, Sinha S: Spontaneous rupture of spinal dermoid cyst with disseminated lipid droplets in central canal and ventricles. J Neurosurg Sci 48:63-65, 2004

7. Gu W, Shang H, Jin X, Xie J, Zhao W: Intradural lumbar mature teratoma with neuronal and glial tissue component in an adult. Neurol Med Chir 50:1112-1115, 2010

8. Jeong SJ, Youm JY, Choi SW, Kim SH: Intradural mature teratoma in the lumbar spine found in adult. Kor J Spine 6:211213, 2009

9. Kalani MY, Iyer S, Coons SW, Smith KA: Spinal intradural teratomas: developmental programs gone awry? Neurosurg Focus 33:E1, 2012

10. Karadag D, Karagulle AT, Erden A, Erden I: MR imaging of a ruptured intraspinal dermoid tumour with fat droplets in the central spinal canal. Australas Radiol 46:444-446, 2002

11. Li Y, Yang B, Song L, Yan D: Mature teratoma of the spinal cord in adults: An unusual case. Oncol Lett 6:942-946, 2013

12. Nickols HH, Chambless LB, Carson RP, Coffin CM, Pearson MM, Abel TW: Intramedullary spinal immature teratoma: resolution of quadriplegia following resection in a 4-week-old infant. J Neurosurg Pediatr 6:586-591, 2010 
13. Oh JS, Im SB, Kim BT, Shin WH: Surgical findings of a lumbar mature teratoma accompanying the preoperative intracranial dissemination of fatty droplets. J Korean Neurosurg Soc 46:409412, 2009

14. Park SC, Kim KJ, Wang KC, Choe G, Kim HJ: Spinal epidural teratoma: Review of spinal teratoma with consideration on the pathogenesis: case report. Neurosurgery 67:1818-1825, 2010
15. Scearce TA, Shaw CM, Bronstein AD, Swanson PD: Intraventricular fat from a ruptured sacral dermoid cyst: clinical, radiographic, and pathological correlation. J Neurosurg 78:666-668, 1993

16. Sung KS, Sung SK, Choi HJ, Song YJ: Spinal intradural extramedullary mature cystic teratoma in an adult. J Korean Neurosurg Soc 44:334-337, 2008 